\title{
Evaluation of the relationship between laboratory parameters and pulmonary function tests in COVID-19 patients
}

\author{
Buğra Kerget ${ }^{1}$, Alperen Aksakal ${ }^{2}$, and Ferhan Kerget ${ }^{2}$ \\ ${ }^{1}$ Ataturk Universitesi \\ ${ }^{2}$ Affiliation not available
}

January 20, 2021

\begin{abstract}
Objective: The novel coronavirus SARS-CoV-2 (COVID-19) rapidly escalated from its origin in an animal market in Wuhan, China in December 2019 to a global pandemic, and the lungs are the most frequently affected organ. The aim of this study was to investigate the relationship between pulmonary function test parameters and laboratory parameters in COVID-19. Method: A total of 60 patients who were admitted to the chest diseases department and intensive care unit of our hospital and were diagnosed with COVID-19 by real-time PCR analysis of nasopharyngeal swabs were evaluated. Pulmonary function tests and laboratory parameters at admission and on day 7 of treatment were analyzed. Results: On day 7 of treatment, white blood cell count, CRP, and fibrinogen level were significantly lower than at admission ( $\mathrm{p}=0.002,0.001$, and 0.001 , respectively), while forced expiratory volume in the first second (FEV1) and forced vital capacity (FVC) values were significantly higher compared to admitting values $(\mathrm{p}=0.001$ for both). Correlation analysis of the changes in respiratory function values and laboratory parameters during follow-up (day 1 to day 7 of treatment) revealed that CRP level was positively correlated with FEV1 $(\mathrm{r}=0.616, \mathrm{p}=0.01)$ and FVC values $(\mathrm{r}=0.51, \mathrm{p}=0.01)$. Fibrinogen level was also positively correlated with $\mathrm{FEV} 1$ ( $\mathrm{r}=0.345$, $\mathrm{p}=0.01)$ and FVC $(\mathrm{r}=0.357, \mathrm{p}=0.01)$. Conclusion: Fibrinogen and CRP levels are easily accessible parameters that may help identify improvement or deterioration in pulmonary function in COVID-19 patients during follow-up and discharge while reducing the risk of transmission.
\end{abstract}

Evaluation of the relationship between laboratory parameters and pulmonary function tests in COVID-19 patients

Short title: Laboratory parameters and pulmonary function tests in COVID-19

Buğra Kerget ${ }^{1}$, Alperen Aksakal ${ }^{2}$, Ferhan Kerget ${ }^{3}$

${ }^{1}$ Depertment of Pulmonary Diseases, Ataturk University School of Medicine, Yakutiye, Erzurum, Turkey

${ }^{2}$ Depertmant of Pulmonary Diseases, Health Sciences University Erzurum Regional Education and Research Hospital, Yakutiye, Erzurum, Turkey

${ }^{3}$ Depertmant of Infection Diseases and Clinical Microbiology, Health Sciences University Erzurum Regional Education and Research Hospital, Yakutiye, Erzurum, Turkey

All authors disclosure no Conflict of Interest.

*Corresponding Author:

Buğra Kerget,MD

Yakutiye Medical Research Center

Chest Disease Department 
25240, Yakutiye

Erzurum, TURKEY

Tel: +904423447446

Fax: +904423446528

bjkerget1903@gmail.com

\title{
Evaluation of the relationship between laboratory parameters and pulmonary function tests in COVID-19 patients
}

\begin{abstract}
Objective: The novel coronavirus SARS-CoV-2 (COVID-19) rapidly escalated from its origin in an animal market in Wuhan, China in December 2019 to a global pandemic, and the lungs are the most frequently affected organ. The aim of this study was to investigate the relationship between pulmonary function test parameters and laboratory parameters in COVID-19.
\end{abstract}

Method : A total of 60 patients who were admitted to the chest diseases department and intensive care unit of our hospital and were diagnosed with COVID-19 by real-time PCR analysis of nasopharyngeal swabs were evaluated. Pulmonary function tests and laboratory parameters at admission and on day 7 of treatment were analyzed.

Results: On day 7 of treatment, white blood cell count, CRP, and fibrinogen level were significantly lower than at admission ( $\mathrm{p}=0.002,0.001$, and 0.001 , respectively), while forced expiratory volume in the first second $\left(\mathrm{FEV}_{1}\right)$ and forced vital capacity $(\mathrm{FVC})$ values were significantly higher compared to admitting values $(\mathrm{p}=0.001$ for both). Correlation analysis of the changes in respiratory function values and laboratory parameters during follow-up (day 1 to day 7 of treatment) revealed that CRP level was positively correlated with $\mathrm{FEV}_{1}(\mathrm{r}=0.616, \mathrm{p}=0.01)$ and $\mathrm{FVC}$ values $(\mathrm{r}=0.51, \mathrm{p}=0.01)$. Fibrinogen level was also positively correlated with $\mathrm{FEV}_{1}(\mathrm{r}=0.345, \mathrm{p}=0.01)$ and $\mathrm{FVC}(\mathrm{r}=0.357, \mathrm{p}=0.01)$.

Conclusion: Fibrinogen and CRP levels are easily accessible parameters that may help identify improvement or deterioration in pulmonary function in COVID-19 patients during follow-up and discharge while reducing the risk of transmission.

Keywords: COVID-19, laboratory parameters, pulmonary function tests

\section{What's already known about this topic?}

It was also reported that pulmonary function tests performed at discharge revealed no significant difference in forced expiratory volume in the first second (FEV1) or forced vital capacity (FVC) values between COVID19 patients with mild, moderate, and severe pneumonia, whereas diffusion capacity decreased in correlation with disease severity. Pulmonary function testing is often not recommended for patients with active COVID19 infection in order to prevent excessive transmission. However, despite clinical improvement in this patient group, ongoing hypoxia and persistent radiological findings cause considerable difficulty in predicting the extent of improvement in pulmonary function.

\section{What does this article add?}

While evaluating the laboratory parameters and radiological findings in patients with COVID-19 has become an easily accessible method, the inability to evaluate the pulmonary function capacities of patients in order to prevent contamination has become the most important problem in follow-up and discharge. Therefore, in our study in which easily accessible parameters were evaluated for guidance, we think that the combined decrease in CRP and fibrinogen levels may be related to increased pulmonary functional capacity.

\section{Introduction}


COVID-19 has infected over 70 million people since it appeared just over a year ago in December 2019, and this number continues to grow daily. The clinical course ranges from asymptomatic or mild (loss of smell and taste, anorexia, muscle and joint pain, fatigue) to severe, particularly in older patients, patients with comorbidities such as hypertension, diabetes mellitus, or chronic kidney disease, pregnant women, and immunocompromised patients ${ }^{1}$.

Acute respiratory failure and macrophage activation syndrome are the most common severe clinical manifestations of COVID-19. Both are characterized by overproduction of proinflammatory cytokines which can lead to endothelial dysfunction in several vital organs, especially the lungs. Recent studies have shown that COVID-19 causes extensive alveolar epithelial destruction, capillary damage/bleeding, hyaline membrane formation, alveolar septal fibrous proliferation, and pulmonary consolidation. Pulmonary function tests have been the most concrete indicator of this lung damage ${ }^{2,3}$.

Studies involving 2-year follow-up of pulmonary function values in patients with severe acute respiratory syndrome (SARS) and Middle East respiratory syndrome (MERS) demonstrated that the main pathology was reduced diffusion capacity (15.5-43.6\%), followed by decreased total lung capacity $(5.2-10.9 \%)^{4,5}$. It was also reported that pulmonary function tests performed at discharge revealed no significant difference in forced expiratory volume in the first second $\left(\mathrm{FEV}_{1}\right)$ or forced vital capacity (FVC) values between COVID-19 patients with mild, moderate, and severe pneumonia, whereas diffusion capacity decreased in correlation with disease severity. Pulmonary function testing is often not recommended for patients with active COVID-19 infection in order to prevent excessive transmission ${ }^{6}$. However, despite clinical improvement in this patient group, ongoing hypoxia and persistent radiological findings cause considerable difficulty in predicting the extent of improvement in pulmonary function.

The aim of this study was to determine the relationship between pulmonary function tests and laboratory parameters for the demonstration of pulmonary dysfunction, which is an important problem for COVID-19 patients.

\section{Materials and Methods}

Study design

The study included patients who presented to the emergency department of Atatürk University with complaints such as recent onset fever, cough, dyspnea, malaise, and sudden loss of taste and smell, and history of international travel or contact with a suspected COVID-19 patient in the last 14 days. Patients (or their relatives) were informed in detail about the aim of the study and all participants provided informed consent forms before being included. Approval was obtained from the local ethics committee prior to study initiation.

Study population

All patients assessed as high risk for COVID-19 underwent high-resolution computed tomography (HRCT) examination. Patients with HRCT findings suggesting COVID-19 and those with atypical radiologic findings but consistent clinical symptoms were admitted. Diagnosis of COVID-19 was based on real-time polymerase chain reaction (PCR) testing of nasopharyngeal swab samples. The study included 60 patients admitted to the Erzurum Regional Training and Research Hospital due to COVID-19 during December 2020 who did not develop acute respiratory distress and macrophage activation syndrome and had a ratio of arterial partial pressure of oxygen to fraction of inspired oxygen $\left(\mathrm{PO}_{2} / \mathrm{FiO}_{2}\right)$ above 200 at admission and on day 7 of treatment.

Starting at admission to the ward, the patients' hematological parameters, biochemical parameters including liver and kidney function tests, coagulation parameters, ferritin, D-dimer, troponin-I, and C-reactive protein (CRP) levels were evaluated daily.

\section{Exclusion criteria}

Patients with any potential contraindications to pulmonary function testing (recent myocardial infarction, pulmonary embolism, cerebral aneurysm, active hemoptysis, pneumothorax, nausea/vomiting, recent tho- 
racic, abdominal, or ocular surgery) were excluded before conducting the tests. In addition, patients who developed acute respiratory distress or macrophage activation syndrome associated with secondary bacterial infections during the 7-day follow-up were also excluded.

Pulmonary function testing

Pulmonary function tests were performed in a negative-pressure room by a technician wearing protective equipment to prevent transmission. The patients' age, height, and weight were measured and recorded. Before testing, patients were instructed to abstain from smoking ( 24 hours), alcohol (4 hours), strenuous exercise (30 minutes), and heavy meals ( 2 hours). Tests were performed with the patients lightly dressed. BTPS correction was performed according to room air and barometric pressure. The technician explained the desired maneuvers to the patients and they performed 3 acceptable spirograms. Tests that met the reproducibility and acceptability criteria were included in our analysis. All spirometry was performed by the same technician using a Plusmed MIR Spirolab III device.

Definitions and Diagnosis

Fever was defined as an axillary temperature of $37.3^{\circ} \mathrm{C}$ or above. Secondary bacterial infection was diagnosed based on signs and symptoms of bacteremia or pneumonia with identification of a new bacterial pathogen in endotracheal aspirate or lower respiratory tract sputum culture. For patients diagnosed as having ventilatorassociated or hospital-acquired pneumonia, treatment was planned according to available guidelines. Acute respiratory distress was diagnosed and graded according to the Berlin 2015 diagnostic criteria. Patients with daily cardiac-specific troponin levels above normal range underwent echocardiographic evaluation for emerging cardiac pathologies. Coagulopathy was defined as prothrombin time $3 \mathrm{~s}$ longer than normal and activated partial thromboplastin time $5 \mathrm{~s}$ longer than normal. Treatment strategy was determined based on disease severity in accordance with the COVID-19 adult diagnosis and treatment guidelines issued by the Turkish Ministry of Health. Macrophage activation syndrome was presumed in patients who despite treatment exhibited findings such as refractory fever, persistently high or increasing CRP and ferritin levels, elevated D-dimer level, lymphopenia or thrombocytopenia, abnormal liver function indicators, hypofibrinogenemia, or elevated triglyceride levels. If daily follow-up of these parameters showed continued deterioration that could not be explained by secondary bacterial infection, $400 \mathrm{mg}$ tocilizumab for macrophage activation syndrome was administered if not contraindicated. Another dose of $400 \mathrm{mg}$ tocilizumab was administered if no significant improvement in laboratory parameters and vital signs was observed after 24 hours.

Biochemical markers

Venous blood samples were obtained from all patients from an antecubital vein after at least 15 min of semisupine rest. Blood samples were collected into tubes containing ethylenediaminetetraacetic acid (EDTA) to prevent coagulation. Troponin-I levels were measured by chemiluminescent immunoassay using an Immulite 2500 device (Siemens Medical Solutions, Erlangen, Germany).

\section{Statistical Analysis}

Data were analyzed using IBM SPSS Statistics version 24.0 software (IBM Corp., Armonk, IL). Categorical variables were expressed as number and percentage; numerical variables were expressed as mean and standard deviation. Kolmogorov-Smirnov test was used to test whether the data were normally distributed. Repeated measures were compared using Friedman test and Wilcoxon test with Bonferroni correction for non-normally distributed numerical data. Pearson correlation analysis was used to assess the relationship between changes in laboratory parameters and changes in pulmonary function tests. Results with $\mathrm{p}$ value $<0.05$ were considered statistically significant.

\section{Results}

The mean age of the patients was $51.4 \pm 18.2$ years. Forty-two (70\%) of the patients were men and 18 (30\%) were women. The mean ages of the male and female patients were $55.1 \pm 12.5$ years and $50.1 \pm 10.4$ years, respectively $(\mathrm{p}=0.04)$. The mean height and weight of the patients were $168.8 \pm 9.1 \mathrm{~cm}$ and $82.3 \pm 11.4 \mathrm{~kg}$, 
respectively. Three of the patients had previously known coronary artery disease, 5 had diabetes mellitus, and 1 had asthma.

The patients' laboratory values and pulmonary function test results at admission and on day 7 of treatment are shown in Table 1. White blood cell count, CRP, and fibrinogen values on day 7 of treatment were significantly lower compared to admitting values $\left(\mathrm{p}=0.002,0.001,0.001\right.$, respectively). $\mathrm{FEV}_{1}$ and $\mathrm{FVC}$ values on day 7 were significantly higher than those at admission ( $\mathrm{p}=0.001$ for both) (Figure 1). Statistical analysis of the patients' pulmonary function values based on the lower limits of normal (LLN) demonstrated that $\mathrm{FEV}_{1}$ and $\mathrm{FVC}$ were significantly reduced at the start of treatment ( $\mathrm{p}=0.05$ for both) and higher but not significantly on day 7 of treatment (Figure 1).

Correlation analysis between the changes in respiratory function values and changes in laboratory parameters during follow-up (day 1 to day 7 of treatment) revealed that CRP level was positively correlated with $\mathrm{FEV}_{1}$ $(\mathrm{r}=0.616, \mathrm{p}=0.01)$ and $\mathrm{FVC}$ values $(\mathrm{r}=0.51, \mathrm{p}=0.01)$ (Figure 2$)$. Fibrinogen level was also positively correlated with $\mathrm{FEV}_{1}(\mathrm{r}=0.345, \mathrm{p}=0.01)$ and $\mathrm{FVC}(\mathrm{r}=0.357, \mathrm{p}=0.01)$ (Figure 3$)$. Among the laboratory parameters, $\mathrm{CRP}$ was found to be correlated with fibrinogen $(\mathrm{r}=0.609, \mathrm{p}=0.01)$ and ferritin $(\mathrm{r}=0.308, \mathrm{p}=0.017)$.

\section{Discussion}

Our study showed that COVID-19 patients' CRP, ferritin, and fibrinogen levels decreased with treatment, consistent with previous studies, while $\mathrm{FEV}_{1}$ and $\mathrm{FVC}$ values increased. The symptoms of restrictive lung disease observed at the start of treatment were improved on day 7 of treatment. Correlation analysis between the 7-day changes in laboratory parameters and pulmonary function tests showed that $\mathrm{FEV}_{1}$ and $\mathrm{FVC}$ values increased in correlation with the change in CRP and fibrinogen levels.

On March 11, 2020, the World Health Organization (WHO) declared the COVID-19 a pandemic and as of December 15, 2020, more than 72 million cases had been confirmed worldwide [1]. Large-scale studies on COVID-19 have documented extensive lung damage both during and after the disease. In a recent report it was determined that COVID-19 pneumonia patients still showed abnormalities in lung CT scans after discharge, with ground-glass opacities being the most common pulmonary sequelae ${ }^{7}$. Survivors of other coronavirus pneumonias (SARS and MERS) are known to have pulmonary function and exercise capacity impairments persisting for months or even years ${ }^{5,8-10}$. To date, however, there have been few studies examining loss of pulmonary function in discharged COVID-19 patients.

SARS-CoV-2 infects the human airway and the vascular and immune cells, potentially leading to severe damage to the respiratory tract, especially within the lungs. Disease severity depends on the effectiveness of the immune system. Current evidence suggests that in some cases, viral infection can cause an exaggerated immune reaction in the host that is severe enough to be classified as the hyperinflammatory condition referred to as macrophage activation syndrome ${ }^{11}$.

Numerous proinflammatory cytokines are released in COVID-19 patients, primarily tumor necrosis factor alpha, interleukins 1, 2, 6 and 18, and nitric oxide. These cytokines can increase vascular permeability, resulting in impaired tissue perfusion, endothelial damage, and microthrombus formation. Increased vascular permeability causes fluid accumulation in the lung tissue and interstitial spaces, which manifests clinically with acute respiratory failure ${ }^{2,3}$. Suppression of these proinflammatory cytokines has proven to be therapeutically beneficial in many inflammatory conditions, including viral infections. Increased cytokine levels in the lung cause alveolar epithelial damage, alveolar septal fibrous proliferation, hyaline membrane formation, and the development of pulmonary consolidation ${ }^{12}$. This is the main cause of the restrictive pathology and reduced diffusion capacity seen in the lungs of COVID-19 patients. Studies have shown that this process continues in association with regression even after discharge ${ }^{13}$. In a study including 110 COVID-19 patients, evaluation of pulmonary function tests at discharge showed a reduction in diffusion capacity that was correlated with disease severity. However, a striking aspect of the study was that the diffusing capacity of the lung for carbon monoxide (DLCO) decreased more than the diffusing capacity divided by alveolar volume (DLCO/VA), suggesting that impairment of the diffusion membrane was a more important issue than low lung volume ${ }^{6}$. 
It was also observed that levels of D-dimer and acute phase reactants such as CRP, ferritin, and fibrinogen were elevated in COVID-19 patients after intense inflammatory cytokine discharge and that these parameters may be associated with morbidity and mortality ${ }^{14}$. Among these parameters, levels of CRP, fibrinogen, and ferritin levels were found to be high and inversely correlated with $\mathrm{FEV}_{1}$ and FVC levels ${ }^{15-17}$. These acute phase reactants are elevated in most inflammatory diseases, and studies evaluating the relationship with parenchymal fibrosis in interstitial lung diseases have demonstrated increases in correlation with the extent of parenchymal fibrosis, highlighting their potential usefulness in follow-up ${ }^{18,19}$.

In the present study, we observed that the restrictive pattern initially presented by COVID-19 patients due to parenchymal infiltration in the lungs resolved during follow-up with regression of the lung consolidation. Pulmonary function values increased with the decline in CRP and fibrinogen levels, suggesting the improvement was secondary to reduced inflammation. However, consistent with our experience with COVID-19, fibrosis may be permanent and patients may show a restrictive pattern with reduced diffusion in long-term follow-up. Plasma fibrogen level has been associated with increased parenchymal fibrosis in previous studies. Therefore, the decline in plasma fibrinogen levels during follow-up in COVID-19 suggests that this parameter may be useful in evaluating progression to parenchymal fibrosis in this disease, which is characterized by pathologies also observed in interstitial lung diseases, such as hyaline membrane formation and alveolar septal fibrous proliferation.

The most important limitation of our study was the inability to evaluate diffusion capacities and total lung capacities when evaluating the etiology of the restrictive pathology observed in pulmonary function tests. However, this was a result of the fact that only portable pulmonary function testing equipment could be placed in the negative pressure room established to minimize the chance of transmission.

In conclusion, although the assessment of laboratory parameters and radiological findings has become a convenient method for evaluating COVID-19 patients, the inability to evaluate patients' pulmonary function due to risk prevention measures has become the biggest problem in follow-up and discharge. In this study examined the guiding potential of some of these easily obtained parameters, we observed that decline in both $\mathrm{CRP}$ and fibrinogen levels may be associated with improved pulmonary functional capacity.

\section{References}

1. Esakandari H, Nabi-Afjadi M, Fakkari-Afjadi J, Farahmandian N, Miresmaeili S-M, Bahreini E. A comprehensive review of COVID-19 characteristics. Biological procedures online. 2020;22:1-10.

2. Kerget B, Kerget F, Koçak AO, et al. Are Serum Interleukin 6 and Surfactant Protein D Levels Associated with the Clinical Course of COVID-19? Lung. 2020;198(5):777-784.

3. Kerget B, Kerget F, Aksakal A, Aşkın S, Sağlam L, Akgün M. Evaluation of alpha defensin, IL-1 receptor antagonist, and IL-18 levels in COVID-19 patients with macrophage activation syndrome and acute respiratory distress syndrome. Journal of Medical Virology. 2020.

4. Ahmed H, Patel K, Greenwood DC, et al. Long-term clinical outcomes in survivors of severe acute respiratory syndrome and Middle East respiratory syndrome coronavirus outbreaks after hospitalisation or ICU admission: a systematic review and meta-analysis. Journal of Rehabilitation Medicine. 2020;52(5).

5. Park WB, Jun KI, Kim G, et al. Correlation between pneumonia severity and pulmonary complications in Middle East respiratory syndrome. Journal of Korean medical science. 2018;33(24).

6. Mo X, Jian W, Su Z, et al. Abnormal pulmonary function in COVID-19 patients at time of hospital discharge. European Respiratory Journal. 2020;55(6).

7. Wang Y, Dong C, Hu Y, et al. Temporal changes of CT findings in 90 patients with COVID-19 pneumonia: a longitudinal study. Radiology. In:2020.

8. Hui D, Joynt G, Wong $\mathrm{K}$, et al. Impact of severe acute respiratory syndrome (SARS) on pulmonary function, functional capacity and quality of life in a cohort of survivors. Thorax. 2005;60(5):401-409. 
9. Hui DS, Wong KT, Ko FW, et al. The 1-year impact of severe acute respiratory syndrome on pulmonary function, exercise capacity, and quality of life in a cohort of survivors. Chest.2005;128(4):2247-2261.

10. Ngai JC, Ko FW, Ng SS, TO KW, Tong M, Hui DS. The long-term impact of severe acute respiratory syndrome on pulmonary function, exercise capacity and health status. Respirology. 2010;15(3):543-550.

11. McGonagle D, Sharif K, O'Regan A, Bridgewood C. Interleukin-6 use in COVID-19 pneumonia related macrophage activation syndrome.Autoimmunity reviews. 2020:102537.

12. Otsuka R, Seino K-i. Macrophage activation syndrome and COVID-19.Inflammation and regeneration. 2020;40(1):1-6.

13. Nusair S. Abnormal carbon monoxide diffusion capacity in COVID-19 patients at time of hospital discharge. European Respiratory Journal. 2020;56(1).

14. Lagadinou M, Solomou EE, Zareifopoulos N, Marangos M, Gogos C, Velissaris D. Prognosis of COVID19: Changes in laboratory parameters. Age (yrs). 2020;62(13.4):47-16.42.

15. Shaaban R, Kony S, Driss F, et al. Change in C-reactive protein levels and FEV1 decline: a longitudinal population-based study.Respiratory medicine. 2006;100(12):2112-2120.

16. Lee CH, Goag EK, Lee SH, et al. Association of serum ferritin levels with smoking and lung function in the Korean adult population: analysis of the fourth and fifth Korean National Health and Nutrition Examination Survey. International journal of chronic obstructive pulmonary disease. 2016;11:3001.

17. Shibata Y, Abe S, Inoue S, et al. Relationship between plasma fibrinogen levels and pulmonary function in the Japanese population: the Takahata study. International journal of medical sciences.2013;10(11):1530.

18. Enomoto N, Oyama Y, Enomoto Y, et al. Prognostic evaluation of serum ferritin in acute exacerbation of idiopathic pulmonary fibrosis. The clinical respiratory journal. 2018;12(8):2378-2389.

19. Bargagli E, Madioni C, Bianchi N, Refini R, Cappelli R, Rottoli P. Serum analysis of coagulation factors in IPF and NSIP.Inflammation. 2014;37(1):10-16.

Figure legends

Figure 1. Comparison of pulmonary function test parameters in COVID-19 patients between admission and day 7 of treatment and with lower limit of normal (LLN) values

Figure 2. Correlation analysis of C-reactive protein level with pulmonary function values

Figure 3. Correlation analysis of fibrinogen level with pulmonary function values

\section{Hosted file}

Table.pdf available at https://authorea.com/users/387698/articles/505002-evaluation-of-therelationship-between-laboratory-parameters-and-pulmonary-function-tests-in-covid-19patients

\section{Hosted file}

Figure.pdf available at https://authorea.com/users/387698/articles/505002-evaluation-ofthe-relationship-between-laboratory-parameters-and-pulmonary-function-tests-in-covid-19patients 